\title{
Enhancement Effect of 3,5-Dichlorophenol on the Solvent Extraction of Scandium(III) with Acetylacetone
}

\author{
Shoichi Katsuta, Hisanori ImURA ${ }^{\dagger}$ and Nobuo Suzuki ${ }^{\dagger \dagger}$ \\ Department of Chemistry, Faculty of Science, Tohoku University, Sendai 980, Japan
}

\begin{abstract}
Keywords Solvent extraction, scandium(III), acetylacetone, 3,5-dichlorophenol, enhancement effect, chelate-proton
\end{abstract} donor association

The synergistic extraction of scandium(III) has been studied in a few systems of thenoyltrifluoroacetone and neutral electron donors, such as tributyl phosphate..$^{1,2}$ These studies have shown that the enhancement effect of electron donors on the chelate extraction of scandium(III) is relatively small. We recently found that the extraction of iron(III) with acetylacetone (Hacac) is remarkably enhanced in the presence of a neutral proton donor, 3,5-dichlorophenol (DCP). ${ }^{3}$ It is very interesting to examine the effect of the proton donor on the chelate extraction of scandium(III).

In the present study, the extraction of scandium(III) with Hacac in heptane was investigated in the presence of DCP. The effect of DCP was quantitatively interpreted by analyzing the extraction equilibrium in detail.

\section{Experimental}

\section{Reagents}

A radioisotope ${ }^{46} \mathrm{Sc}$, Hacac and heptane were either prepared or purified as described previously. ${ }^{4}$ DCP of 99\% purity was purchased, and then further purified by vacuum sublimation. Water was doubly distilled. The other reagents were of analytical reagent grade and used as obtained.

\section{Procedure}

An aqueous solution containing $0.10 \mathrm{M}$ sodium perchlorate and $1.0 \times 10^{-5} \mathrm{M}\left(\mathrm{M}=\mathrm{mol} \mathrm{dm}^{-3}\right)$ scandium(III) labeled with ${ }^{46} \mathrm{Sc}$ was mechanically shaken with an equal volume of a heptane solution containing $5.1 \times$ $10^{-3}-5.1 \times 10^{-2} \mathrm{M}$ Hacac and $7.2 \times 10^{-4}-5.0 \times 10^{-2} \mathrm{M} \mathrm{DCP}$ for $15-120 \mathrm{~min}$ at $25^{\circ} \mathrm{C}$, and then centrifuged. The $\gamma$-activities of both phases were measured with a well-type $\mathrm{NaI}(\mathrm{Tl})$ scintillation counter and the distribution ratio $(D)$ of scandium(III) was calculated. The $\mathrm{pH}$ value of

$\dagger$ Present address: Department of Chemistry, Faculty of Science, Ibaraki University, Mito 310, Japan.

t† To whom correspondence should be addressed. the aqueous phase was adjusted with $1 \times 10^{-4}-1 \times 10^{-3} \mathrm{M}$ perchloric acid, acetic acid and sodium hydroxide solutions and measured with a glass electrode after shaking.

\section{Results and Discussion}

The extraction of scandium(III) with $0.010 \mathrm{M}$ Hacac in either the absence or presence of $0.050 \mathrm{M} \mathrm{DCP}$ in heptane was carried out at various $\mathrm{pH}$. The results are shown in Fig. 1. Although scandium(III) can not be extracted quantitatively with Hacac alone, the addition of $0.050 \mathrm{M}$ DCP enhances the distribution ratio of scandium(III) by about 200 -fold over the entire $\mathrm{pH}$

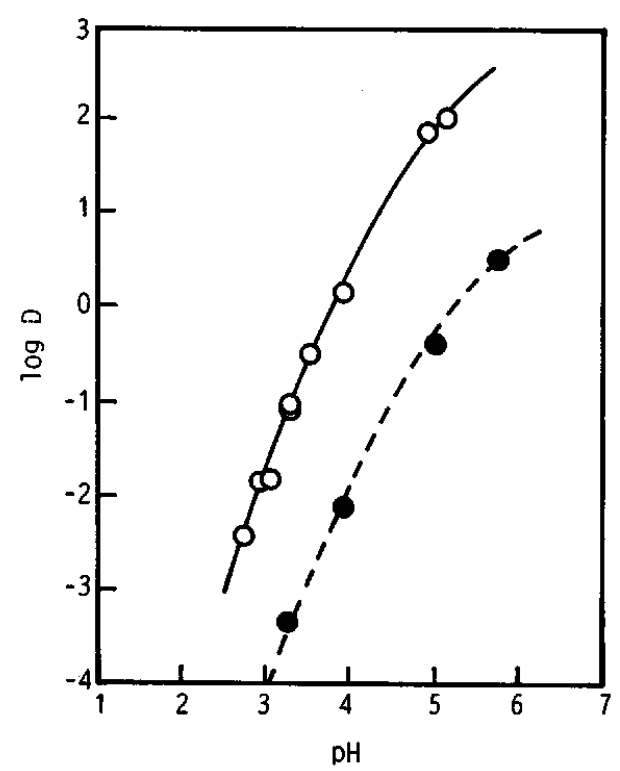

Fig. 1 Effect of DCP on the extraction of scandium(III) with Hacac in heptane. Initial composition of the organic phase: $1.0 \times 10^{-2} \mathrm{M}$ Hacac; $\mathrm{O} 1.0 \times 10^{-2} \mathrm{M}$ Hacac and $5.0 \times 10^{-2} \mathrm{M}$ DCP. 
region studied; the percent extraction reaches to $99 \%$ at pH 5. This effect of DCP is larger in magnitude than the enhancement effect of the neutral electron donors on the extraction of scandium(III) with thenoyltrifluoroacetone. ${ }^{1,2}$ It was confirmed that scandium(III) is not extracted at all with DCP alone.

\section{Extraction equilibrium}

The distribution ratio of scandium(III) with Hacac alone $\left(D_{0}\right)$ can be expressed as

$$
\begin{aligned}
D_{0} & =\frac{\left[\overline{\mathrm{Sc}(\mathrm{acac})_{3}}\right]}{\left[\mathrm{Sc}^{3+}\right]+\sum_{j}\left[\mathrm{Sc}(\mathrm{acac}) j^{\left(3^{-j j+}+\right.}\right]} \\
& =P_{\mathrm{M}} \beta_{3}\left[\mathrm{acac}^{-}\right]^{3}\left(1+\sum_{j} \beta_{j}\left[\mathrm{acac}^{-}\right]^{j}\right)^{-1},
\end{aligned}
$$

where the bar denotes the organic phase, acac the acetylacetonate anion, $P_{M}$ the partition coefficient of $\mathrm{Sc}(\mathrm{acac})_{3}$, and $\beta_{j}$ the overall formation constant of $\mathrm{Sc}(\mathrm{acac}) j^{(3-j)+}$, respectively. These equilibrium constants were previously determined, i.e., $\log P_{\mathrm{M}}=1.041, \log \beta_{1}=$ 8.02, $\log \beta_{2}=14.38$, and $\log \beta_{3}=19.39 .^{4}$

In our previous studies, it was shown that $\mathrm{Cr}$ (acac) ${ }_{3}$ and $\mathrm{Co}(\mathrm{acac})_{3}$ form association complexes with halogenated phenols in nonpolar organic solvents by hydrogen bonding. ${ }^{5,6}$ The enhanced extraction of scandium(III) by DCP is therefore attributable to the association of $\mathrm{Sc}(\mathrm{acac})_{3}$ with DCP in the organic phase: the distribution ratio of scandium(III) with Hacac in the presence of DCP $(D)$ can be expressed as

$$
\begin{aligned}
D & =\frac{\left[\overline{\mathrm{Sc}(\mathrm{acac})_{3}}\right]+\sum_{n}\left[\overline{\mathrm{Sc}(\mathrm{acac})_{3} \cdot n \overline{\mathrm{DCP}}}\right]}{\left[\mathrm{Sc}^{3+}\right]+\sum_{j}\left[\mathrm{Sc}(\mathrm{acac})_{j}^{(3-j)+}\right]} \\
& =D_{0}\left(1+\sum_{n} \beta_{\mathrm{ass}, n}[\overline{\mathrm{DCP}}]^{n}\right),
\end{aligned}
$$

where $\beta_{\text {ass }, n}$ is the association constant in the organic phase, corresponding to the following equilibrium:

$$
\begin{aligned}
& \overline{\mathrm{Sc}(\mathrm{acac})_{3}}+n \overline{\mathrm{DCP}} \rightleftharpoons \overline{\mathrm{Sc}(\mathrm{acac})_{3} \cdot n \mathrm{DCP}}, \\
& \beta_{\mathrm{ass}, n}=\left[\overline{\mathrm{Sc}(\mathrm{acac})_{3} \cdot n \overline{\mathrm{DCP}}}\right]\left[\overline{\mathrm{Sc}(\mathrm{acac})_{3}}\right]^{-1}[\overline{\mathrm{DCP}}]^{-n} .
\end{aligned}
$$

Therefore,

$$
D / D_{0}=1+\sum_{n} \beta_{\text {ass },[}[\overline{\mathrm{DCP}}]^{n} .
$$

The $\beta_{\text {ass }, n}$ value can be determined based on Eq. (5) if reliable values of $D_{0}$ and the equilibrium DCP concentration in the organic phase $([\overline{\mathrm{DCP}}])$ are obtained. The estimation of $D_{0}$ and $[\overline{\mathrm{DCP}}]$ should be made by considering the association between Hacac and DCP in the organic phase:

$$
\begin{aligned}
& \overline{\mathrm{Hacac}}+i \overline{\mathrm{DCP}} \rightleftharpoons \overline{\mathrm{Hacac} \cdot i \mathrm{DCP}} \\
& \beta_{\text {ass } i}^{*}=[\overline{\mathrm{Hacac} \cdot i \mathrm{DCP}}][\overline{\mathrm{Hacac}}]^{-1}[\overline{\mathrm{DCP}}]^{-i},
\end{aligned}
$$

where the association constant of Hacac with DCP,

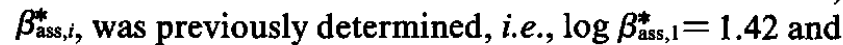
$\log \beta_{\text {ass }, 2}^{*}=2.66 .^{3}$ The $[\overline{\mathrm{DCP}}]$ and $[\overline{\mathrm{Hacac}}]$ values were computed by solving the following simultaneous equations derived from the mass balances for Hacac and DCP:

$$
\begin{aligned}
C_{\mathrm{AA}}= & \left\{\left(1+K_{\mathrm{a}, \mathrm{AA}} /\left[\mathrm{H}^{+}\right]\right) / P_{\mathrm{AA}}+1+\beta_{\mathrm{ass}, 1}^{*}[\overline{\mathrm{DCP}}]\right. \\
& \left.+\beta_{\mathrm{ass}, 2}^{*}[\overline{\mathrm{DCP}}]^{2}\right\}[\overline{\mathrm{Hacac}}]
\end{aligned}
$$

and

$$
\begin{aligned}
C_{\mathrm{CP}}= & \left\{\left(1+K_{\mathrm{a}, \mathrm{CP}} /\left[\mathrm{H}^{+}\right]\right) / P_{\mathrm{CP}}+1+\beta_{\mathrm{ass}, 1}^{*}[\overline{\mathrm{Hacac}}]\right\}[\overline{\mathrm{DCP}}] \\
& +2 \beta_{\mathrm{ass}, 2}^{*}[\overline{\mathrm{Hacac}}][\overline{\mathrm{DCP}}]^{2}
\end{aligned}
$$

where $C, P$, and $K_{\mathrm{a}}$ denote the initial concentration, partition coefficient, and acid-dissociation constant, respectively, and subscripts $\mathrm{AA}$ and $\mathrm{CP}$ refer to Hacac and DCP. The literature values of $P$ and $K_{\mathrm{a}}$ were adopted, i.e., $P_{\mathrm{AA}}=0.887,{ }^{7} P_{\mathrm{CP}}=2.61,{ }^{8} \mathrm{p} K_{\mathrm{a}, \mathrm{AA}}=8.82,{ }^{9}$ and $\mathrm{p} K_{\mathrm{a}, \mathrm{CP}}=8.25 .{ }^{10}$ The $D_{0}$ value can be obtained from Eq. (1) using [acac $\left.{ }^{-}\right]$calculated as $K_{\mathrm{a}, \mathrm{AA}}[\overline{\mathrm{Hacac}}] P_{\mathrm{AA}}^{-1} \times$ $\left[\mathrm{H}^{+}\right]^{-1}$.

Figure 2 shows a plot of $\log \left(D / D_{0}\right)$ vs. $\log [\overline{\mathrm{DCP}}]$. The slope of the plot becomes close to the limiting value of 3.0 in the higher concentration region of DCP. This means that the maximum number of DCP molecules associated with one molecule of $\mathrm{Sc}(\mathrm{acac})_{3}$ is 3 under these

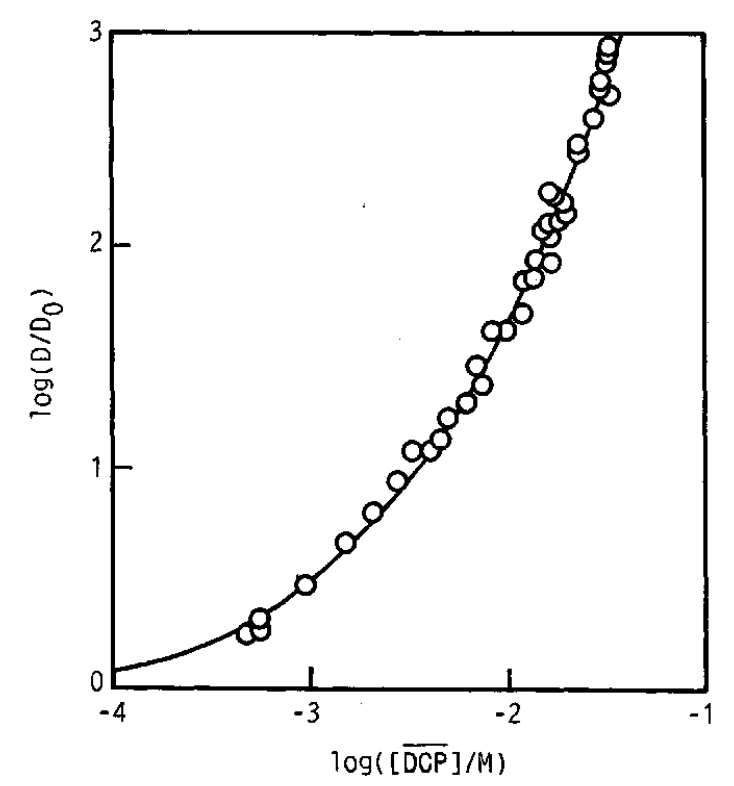

Fig. $2 D / D_{0}$ as a function of the equilibrium DCP concentration in the organic phase. Initial composition of the organic phase: $5.1 \times 10^{-3}-5.1 \times 10^{-2} \mathrm{M}$ Hacac and $7.2 \times 10^{-4}$ $5.0 \times 10^{-2} \mathrm{M}$ DCP. Equilibrium $\mathrm{pH}: 2.8-3.9$. $D_{0}$ and $[\overline{\mathrm{DCP}}]$ values are calculated by considering the Hacac-DCP association (see the text). The solid line indicates the regression curve based on Eq. (5). 
experimental conditions. The plot was treated by a nonlinear least-squares method (SALS program, The University of Tokyo) based on Eq. (5), and the association constants, $\log \beta_{\text {ass }, 1}=3.30 \pm 0.05, \log \beta_{\text {ass }, 2}=$ $5.04 \pm 0.23$ and $\log \beta_{\text {ass }, 3}=7.16 \pm 0.08$, were obtained. These association constants are comparable to those of $\mathrm{Fe}(\mathrm{acac})_{3}$ with $\mathrm{DCP}$ in heptane, i.e., $\log \beta_{\mathrm{ass}, 1}=3.41$, $\log \beta_{\text {ass }, 2}=5.97$ and $\log \beta_{\text {ass }, 3}=7.50 .^{3}$

The $\beta_{\text {ass, } 1}$ of Sc(acac) $)_{3}$ with DCP is also comparable to the $1: 1$ adduct-formation constants of tris(thenoyltrifluoroacetonato)scandium(III) ( $\mathrm{Sc}(\mathrm{tta})_{3}$ ) with the neutral electron donors so far reported.1,2 However, Sc(acac) can form highly stable $1: 2$ and $1: 3$ association complexes with DCP, whereas $\mathrm{Sc}(\mathrm{tta})_{3}$ can only form the $1: 1$ adduct with the electron donors. Consequently, DCP can exert a larger enhancement effect on the chelate extraction of scandium(III) than the electron donors, thus enabling a sensitive extraction control by the concentration of DCP.

\section{References}

1. T. Sekine and D. Dyrssen, J. Inorg. Nucl. Chem., 29, 1481
(1967).

2. K. Akiba, T. Ishikawa and N. Suzuki, J. Inorg. Nucl. Chem., 33, 4161 (1971).

3. S. Katsuta, H. Imura and N. Suzuki, J. Radioanal. Nucl. Chem., submitted for publication.

4. N. Suzuki, J. Kodera and H. Imura, Inorg. Chim. Acta, 128, 261 (1987).

5. S. Katsuta, H. Imura and N. Suzuki, Chem. Lett., 1991, 733.

6. S. Katsuta, H. Imura and N. Suzuki, Bull. Chem. Soc. Jpn., in press.

7. H. Watarai and N. Suzuki, Bull. Chem. Soc. Jpn., 52, 2778 (1979).

8. S. Katsuta, M. Sc. Thesis, Tohoku University, 1989.

9. J. Rydberg, Svensk Kem. Tidskr., 65, 37 (1953); [Chem. Abstr., 47, 10968h (1953)].

10. J. Drahonovsky and Z. Vacek, Collect. Czech. Chem. Commun., 36, 3431 (1971).

(Received April 8, 1991)

(Accepted June 28, 1991) 